\title{
Biogenic selenium and tellurium nanoparticles synthesized by environmental microbial isolates efficaciously inhibit bacterial planktonic cultures and biofilms
}

\author{
Emanuele Zonaro ${ }^{1,2 *}$, Silvia Lampis ${ }^{1}$, Raymond J. Turner ${ }^{2}$, S. Junaid S. Qazi ${ }^{2}$ and \\ Giovanni Vallini ${ }^{1 *}$ \\ ${ }^{1}$ Department of Biotechnology, University of Verona, Verona, Italy, ${ }^{2}$ Biofilm Research Group, Department of Biological \\ Sciences, University of Calgary, Calgary, Canada
}

OPEN ACCESS

Edited by:

David W. Graham

Newcastle University, UK

Reviewed by:

Marco Rinaldo Oggioni,

University of Leicester, UK

Jay Nadeau,

McGill University, Canada

*Correspondence: Emanuele Zonaro and Giovanni Vallini,

Department of Biotechnology, University of Verona, Strada Le Grazie

15, 37134 Verona, Italy emanuele.zonaro@univr.it;

giovanni.vallini@univr.it

Specialty section:

This article was submitted to Antimicrobials, Resistance and

Chemotherapy,

a section of the journal

Frontiers in Microbiology

Received: 05 February 2015 Accepted: 27 May 2015

Published: 16 June 2015

Citation:

Zonaro E, Lampis S, Turner RJ, Qazi SJS and Vallini G (2015) Biogenic selenium and tellurium nanoparticles

synthesized by environmental microbial isolates efficaciously inhibit bacterial planktonic cultures and biofilms. Front. Microbiol. 6:584 doi: 10.3389/fmicb.2015.00584
The present study deals with $\mathrm{Se}^{0}$ - and $\mathrm{Te}^{0}$-based nanoparticles bio-synthesized by two selenite- and tellurite-reducing bacterial strains, namely Stenotrophomonas maltophilia SelTE02 and Ochrobactrum sp. MPV1, isolated from polluted sites. We evidenced that, by regulating culture conditions and exposure time to the selenite and tellurite oxyanions, differently sized zero-valent Se and Te nanoparticles were produced. The results revealed that these $\mathrm{Se}^{0}$ and $\mathrm{Te}^{0}$ nanoparticles possess antimicrobial and biofilm eradication activity against Escherichia coli JM109, Pseudomonas aeruginosa PAO1, and Staphylococcus aureus ATCC 25923. In particular, $\mathrm{Se}^{0}$ nanoparticles exhibited antimicrobial activity at quite low concentrations, below that of selenite. Toxic effects of both $\mathrm{Se}^{0}$ and $\mathrm{Te}^{0}$ nanoparticles can be related to the production of reactive oxygen species upon exposure of the bacterial cultures. Evidence so far achieved suggests that the antimicrobial activity seems to be strictly linked to the dimensions of the nanoparticles: indeed, the highest activity was shown by nanoparticles of smaller sizes. In particular, it is worth noting how the bacteria tested in biofilm mode responded to the treatment by $\mathrm{Se}^{0}$ and $\mathrm{Te}^{0}$ nanoparticles with a susceptibility similar to that observed in planktonic cultures. This suggests a possible exploitation of both $\mathrm{Se}^{0}$ and $\mathrm{Te}^{0}$ nanoparticles as efficacious antimicrobial agents with a remarkable biofilm eradication capacity.

Keywords: antimicrobial activity, bacterial biosynthesis, biofilm growth mode, nanoparticles, selenium, tellurium

\section{Introduction}

In the last few decades, the emergence of bacterial resistance to antibiotics has become a common threat in both hospitals and community settings. As a consequence, the effectiveness of antibiotic treatment of bacterial infections has progressively decreased (Sievert et al., 2013). The problem is particularly relevant in the treatment of biofilm-associated infections, since bacteria grown in biofilm mode are more tolerant to conventional antibiotics and biocides compared to free swimming cells (Stewart and Costerton, 2001). Therefore, it is becoming increasingly imperative to develop and test new antimicrobial compounds capable of bactericidal activity even in biofilm growth mode toward multidrug resistant bacterial species. 
In recent years, the utilization of metal ions and metal nanoparticles has emerged as an alternative to the use of organic compounds as antimicrobial agents (Lemire et al., 2013). Indeed, a widespread antimicrobial activity is often a common trait of nanomaterials, mainly due to the high surface to volume ratio of their constituent particles which results in a high reactivity. A vast literature exists dealing with the antimicrobial activity of silver nanoparticles (Sondi and Salopek-Sondi, 2004; Fabrega et al., 2009; Martinez-Gutierrez et al., 2013): however other metal or metalloid nanoparticles have shown so far bactericidal effects. For instance, the antimicrobial activity of both $\mathrm{ZnO}$ (Jones et al., 2008) and $\mathrm{TiO}_{2}$ (Tsuang et al., 2008) nanoparticles has been reported. In addition, selenium- (Tran and Webster, 2011) and tellurium-based (Mohanty et al., 2014) nanomaterials have revealed interesting antimicrobial potential against a broad range of pathogenic strains. This opens a new perspective for these nanoparticles in terms of coating agents in medical devices and health-related products to prevent bacterial infections (Roe et al., 2008). Moreover, they can find promising applications also in industrial settings as a potential tool to contrast biofouling (Zhang et al., 2012).

Nevertheless, currently the prominent drawback to the use of metal nanoparticles is the cost associated with their synthesis by means of traditional physico-chemical methods with production of toxic by-products (Narayanan and Sakthivel, 2010). For these reasons, nowadays a growing interest exists in developing new eco-friendly processes for the synthesis of potentially valuable nanoparticles. Among the possible alternatives, biogenesis represents an interesting option through the exploitation of the microbial ability to change the oxidation state of metal/metalloid salts with the final production of nanoparticles (Narayanan and Sakthivel, 2010). Such a green production of nanomaterials seems particularly suitable for the synthesis of stable monodisperse nanoparticles.

In this study we focused on the potential antimicrobial activity and biofilm eradication effect of zero-valent selenium and tellurium nanoparticles of biogenic origin on cultures of three biofilm-forming bacterial isolates, namely Escherichia coli JM109, Pseudomonas aeruginosa PAO1, and Staphylococcus aureus ATCC 25923. Experiments were carried out to test both the antimicrobial activity against either planktonic cells or cultures grown in biofilm mode and a possible involvement of reactive oxygen species as mechanism of toxicity of $\mathrm{Se}^{0}$ and $\mathrm{Te}^{0}$ nanoparticles toward the bacterial strains considered.

\section{Materials and Methods}

\section{Preparation of Biogenic Se and Te Nanoparticles}

Two environmental isolates, Stenotrophomonas maltophilia SeITE02 (Di Gregorio et al., 2005) and Ochrobactrum sp. MPV1 (Santi, 2013) were used to produce respectively Se and Te NPs. Sterile Nutrient Broth supplemented with $0.5 \mathrm{mM} \mathrm{Na}_{2} \mathrm{SeO}_{3}$ or $0.3 \mathrm{mM} \mathrm{K}_{2} \mathrm{TeO}_{3}$ was inoculated with $1 \mathrm{~mL}$ of fresh inoculum. The culture was then incubated aerobically at $27^{\circ} \mathrm{C}$. Bacterial cells and NPs were removed from culture medium after different time by centrifugation at $10,000 \times \mathrm{g}$ for $10 \mathrm{~min}$. The pellets were washed twice with $0.9 \% \mathrm{NaCl}$ solution, resuspended in Tris/ $\mathrm{HCl}$ buffer ( $\mathrm{pH}$ 8.2) and cells were then disrupted by ultrasonication at $100 \mathrm{~W}$ for $5 \mathrm{~min}$. The suspension was then centrifuged at $10,000 \times \mathrm{g}$ for $30 \mathrm{~min}$ to separate disrupted cells (pellet) from NPs (supernatant). NPs were recovered after centrifugation at $40,000 \times \mathrm{g}$ for $30 \mathrm{~min}$, washed twice and resuspended in deionized water.

\section{Dynamic Light Scattering (DLS) Analysis}

Measurements of Dynamic Light Scattering (DLS) from dispersed nanoparticles were made in Nanoscience lab at University of Calgary. Data were measured using a Zetasizer Nano-ZS by Malvern instrument with $\mathrm{He}-\mathrm{Ne}$ laser at the wavelength of $633 \mathrm{~nm}$ and a power of $4.0 \mathrm{~mW}$ as a light source collecting data at a fixed scattering angle of $173^{\circ} .300 \mu \mathrm{L}$ of the sample was applied to a quartz cell with a $10 \mathrm{~mm}$ path length and data collected at $25^{\circ} \mathrm{C}$. From the autocorrelation function, the relaxation rate, $\Gamma$, is determined allowing for the translational diffusion coefficient, $\mathrm{D}$, to be calculated using $\mathrm{D}=\Gamma / \mathrm{Q} 2$, where $\mathrm{Q}$ in the magnitude of the scattering vector $[\mathrm{Q}=(4 \pi \mathrm{n} / \lambda) \sin \theta$; $\mathrm{n}$ is the refractive index of the solution, $\lambda$ the wavelength of the scattered light, and $\theta$ the scattering angle]. The viscosity of the water was taken as $8.9 \times 10^{-4} \mathrm{~Pa}$ s and its refractive index as 1.33 at $25^{\circ} \mathrm{C}$. The diffusion coefficients of the dispersed particles can be determined from the intensity of the autocorrelation function. Hydrodynamic diameter, $\mathrm{Dh}$, can then be calculated from the diffusion coefficients, $\mathrm{D}$, by using the Stokes-Einstein relation $[\mathrm{Dh}=(\mathrm{KBT}) / 3 \pi \eta \mathrm{D}$; where $\mathrm{KBT}$ in thermal energy and $\eta$ is the viscosity of the dispersion medium]. Our analysis used a cumulant fit to the correlation function and gives the averaged weighted diameter and a polydispersity index (PDI). A regularized fit to the DLS data gives more details on the size distribution of the dispersed nanoparticles. All the values were obtained using the software provided by the Malvern with the instrument.

\section{Scanning Electron Microscpy (SEM) Analysis}

The morphology and elemental composition of the purified Se- and Te-NPs were analyzed through scanning electron microscopy (SEM). Nanoparticles were fixed, dehydrated with increasing ethanol concentrations and dried through the critical point method by using liquid $\mathrm{CO}_{2}$. They were mounted on metallic specimens stubs and then directly observed through XL30 ESEM (FEI-Philips) equipped with an EDAX microanalytical system.

\section{Evaluation of MIC (Minimun Inhibitory Concentration)}

Minimum inhibitory concentration of Se and $\mathrm{Te}-\mathrm{NPs}_{6 \mathrm{H}}$ was evaluated against the following five different strains: $E$. coli JM109, E. coli ATCC 25922, $P$. aeruginosa PAO1, $P$. aeruginosa ATCC 27853, and S. aureus ATCC 25923. Briefly, in a 96well microtitre plate, wells were inoculated with $75 \mu \mathrm{L}$ of $1 / 15$ dilution of a 1.0 McFarland standard, LB broth and increasing concentrations of NPs $(7.8125,15.6250,31.25,62.5,125,250$, $500,1000,2000,4000,8000 \mathrm{mg} / \mathrm{L})$. After $24 \mathrm{~h}$ of incubation at $37^{\circ} \mathrm{C}$, cultures were serially diluted and spot plated onto Nutrient Broth agar plates. After $48 \mathrm{~h}$ of incubation at $37^{\circ} \mathrm{C}$, viability was 
quantified by counting colonies. The MIC values were recorded as the lowest concentrations of NPs that completely inhibited bacterial growth of the different strains tested.

\section{Antimicrobial Activity Evaluation}

The antimicrobial activity of the different nanoparticles produced was evaluated against three reference strains: E. coli JM109, P. aeruginosa PAO1, and S. aureus ATCC 25923. A modification of the MBEC $^{\mathrm{TM}}$ protocol (Harrison et al., 2010) was utilized. In a 96-well microtitre plate, all wells were inoculated with $75 \mu \mathrm{L}$ of $1 / 15$ dilution of a $1.0 \mathrm{McF}$ arland standard and $75 \mu \mathrm{L}$ of LB broth. The MBEC $^{\mathrm{TM}}$ lid was then attached and cultures were incubated $37^{\circ} \mathrm{C}, 95 \%$ humidity, shaken at $125 \mathrm{rpm}$. After $48 \mathrm{~h}$ of growth to establish the biofilms, the MBEC $^{\mathrm{TM}}$ lid was moved and exposed to another 96-well microtitre plate, containing an increasing gradient of nanoparticles concentrations. The viability of the bacterial cultures was evaluated using the established MBEC $^{\mathrm{TM}}$ recovery protocol (Harrison et al., 2010) after $4 \mathrm{~h}$ of exposure, to test the ability of the nanoparticles to inhibit biofilm formation, and $24 \mathrm{~h}$ of exposure after $24 \mathrm{~h}$ of growth, to evaluate the biofilm eradication capability.

Bioflms were recovered by rinsing MBEC lid twice in $200 \mu \mathrm{L}$ saline for $1 \mathrm{~min}$ and sonicating for $10 \mathrm{~min}$ at $60 \mathrm{~Hz}$ in a 96-well microtitre plate containing $200 \mu \mathrm{L}$ Nutrient Broth, $0.1 \%$ Tween-20 and $1 / 50$ diluted universal neutralizer. The planktonic cultures were also recovered by adding $10 \mu \mathrm{L}$ of a $1 / 10$ dilution of universal neutralizer to $40 \mu \mathrm{L}$ of cultures. Both biofilm and planktonic cultures were serially diluted and spot plated onto Nutrient Broth agar plates. After $48 \mathrm{~h}$ of incubation at $37^{\circ} \mathrm{C}$, viability was quantified by counting colonies.

The effective concentration causing $50 \%$ growth inhibition $\left(\mathrm{EC}_{50}\right)$ was calculated with GraphPad Prism 6.01 software. All tests were carried out in triplicate $(n=3)$ and the results were averaged.

\section{Confocal Laser Scanning Microscopy (CSLM) Analysis}

Biofilms were visualized by CLSM using both the fluorescent stain Acridine Orange (AO), the LIVE/DEAD ${ }^{\circledR}$ BacLight $^{\mathrm{TM}}$ Bacterial Viability Kits and a Leica DM IRE2 microscope with a $64 \times$ water immersion objective. $3 \mathrm{D}$ images were generated using Imaris x64 Image Processing Software (Bitplane Scientific Software, South Windsor, CT, USA).

\section{Quantification of Reactive Oxygen Species (ROS)}

The ROS formed after the exposition of bacterial culture to SeNPs or TeNPs were quantified using $2^{\prime}-7^{\prime}$ dichlorofluoresceindiacetate (DCFDA) (Wang and Joseph, 1999). The concentration of nanoparticles used was $30 \mathrm{mg} / \mathrm{L}$. DCFDA interaction with NPs alone was tested. Cultures were incubated at $37^{\circ} \mathrm{C}$ for $3 \mathrm{~h}$ and then centrifuged at $4^{\circ} \mathrm{C}$ for $30 \mathrm{~min}$ at $300 \times \mathrm{g}$. DCFDA was then added to the supernatant at a concentration of $100 \mu \mathrm{M}$. After $1 \mathrm{~h}$, ROS were quantified at $485 / 20 \mathrm{~nm}$ of fluorescence excitation wavelength and $528 / 20 \mathrm{~nm}$ of emission wavelength using Gemini EM Fluorescence Microplate Reader (MolecularDevices, U.S.A.).
Differences between the ROS production induced by the different nanoparticles were determined using One-Way analysis of variance (ANOVA). The level of significance was set at $P<0.05$. All tests were carried out in triplicate $(n=3)$ and the results were averaged.

\section{Results}

\section{Biogenesis and Characterization of Se and $\mathrm{Te}$ Nanoparticles}

Different types of NPs were produced using Stenotrophomonas maltophilia SeITE02 and Ochrobactrum sp. MPV1. The two strains had previously shown the ability to reduce respectively, selenite (Di Gregorio et al., 2005) and tellurite to the elemental forms. NPs were produced after $6 \mathrm{~h}$ of bacteria incubation with the oxyanions $\left(\mathrm{SeNPs}_{6 \mathrm{H}}\right.$ and $\left.\mathrm{TeNPs}_{6 \mathrm{H}}\right), 24 \mathrm{~h}$ of incubation $\left(\mathrm{SeNPs}_{24 \mathrm{H}}\right.$ and $\left.\mathrm{TeNPs}_{24 \mathrm{H}}\right)$, and $48 \mathrm{~h}\left(\mathrm{SeNPs}_{48 \mathrm{H}}\right.$ and $\left.\mathrm{TeNPs}_{48 \mathrm{H}}\right)$.

SEM observations indicate that both $\mathrm{Se}$ and $\mathrm{Te}$ are well dispersed and of spherical shape (Figure 1).

EDX microanalysis of the purified SeNPs and TeNPs exhibited respectively the characteristic Se absorption peaks at 1.37, 11.22 , and $12.49 \mathrm{keV}$ and the Te absorption peak at $3.769 \mathrm{keV}$. Moreover, EDX analysis revealed also the presence of the absorption peaks of $\mathrm{C}, \mathrm{O}, \mathrm{P}$, and $\mathrm{S}$, indicating the possible association of the purified $\mathrm{Se}$ and Te NPs with organic compounds.

The different kinds of NPs produced were also characterized by using dynamic light scattering (DLS) technique (Figure 2). For what concerns SeNPs, the diameters of the NPs increase with the incubation times. After $6 \mathrm{~h}$ of incubation, the average hydrodynamic diameter of the NPs, calculated through cumulant fit, is $221.1 \mathrm{~nm}$, with a polydispersity index (PDI) of 0.24 . As the incubation time increases to 24 and $48 \mathrm{~h}$, also the diameter of the NPs grows to values of $345.2 \mathrm{~nm}(\mathrm{PDI}=0.21)$ and $357.1 \mathrm{~nm}$ (PDI of 0.24 ), respectively. On the other hand, the diameter of TeNPs remains almost stable as the incubation time increases, with values of $78.5 \mathrm{~nm}$ (PDI $=0.50)$ after $6 \mathrm{~h}$ of incubation, $76.2 \mathrm{~nm}(\mathrm{PDI}=0.49)$ after $24 \mathrm{~h}$ and $76.1 \mathrm{~nm}(\mathrm{PDI}=0.50)$ after $48 \mathrm{~h}$.

\section{Evaluation of MIC Values}

MIC values obtained with Se and Te-NPs 6 for E. coli JM109, E. coli ATCC 25922, $P$. aeruginosa PAO1, P. aeruginosa ATCC 27853 and S. aureus ATCC 25923 are shown in Table 1. The values with $\mathrm{SeNPs}_{24 \mathrm{H}}$ were $125 \mathrm{mg} / \mathrm{L}$ for E.coli JM109 and $E$. coli ATCC 25922 while $250 \mathrm{mg} / \mathrm{L}$ for $P$. aeruginosa PAO1, $P$. aeruginosa ATCC 27853 and S. aureus ATCC 25923. On the other hand, the value with $\mathrm{TeNPs}_{6 \mathrm{H}}$ was $500 \mathrm{mg} / \mathrm{L}$ for all strains tested, except for the test with $S$. aureus ATCC 25923 in which a MIC of $1000 \mathrm{mg} / \mathrm{L}$ was registered.

\section{Evaluation of Antimicrobial and Antibiofilm Activity of Nanoparticles}

The susceptibility of E. coli JM109, P. aeruginosa PAO1 and S. aureus ATCC 25923 biofilm and planktonic population to the different biogenic NPs was evaluated. Analyses were performed on only one strain for each bacterial species considered. The 

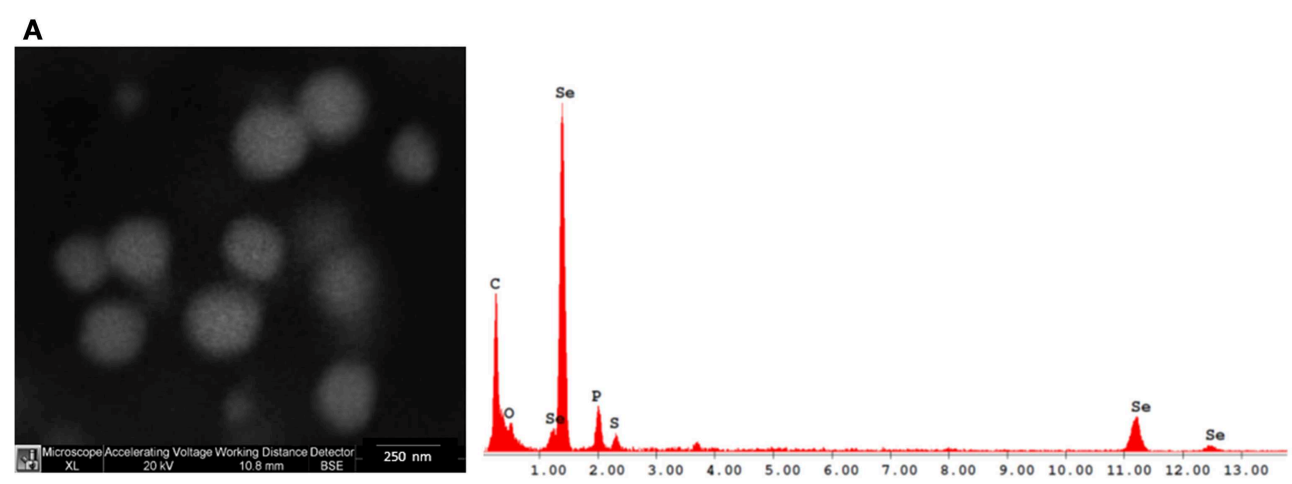

B
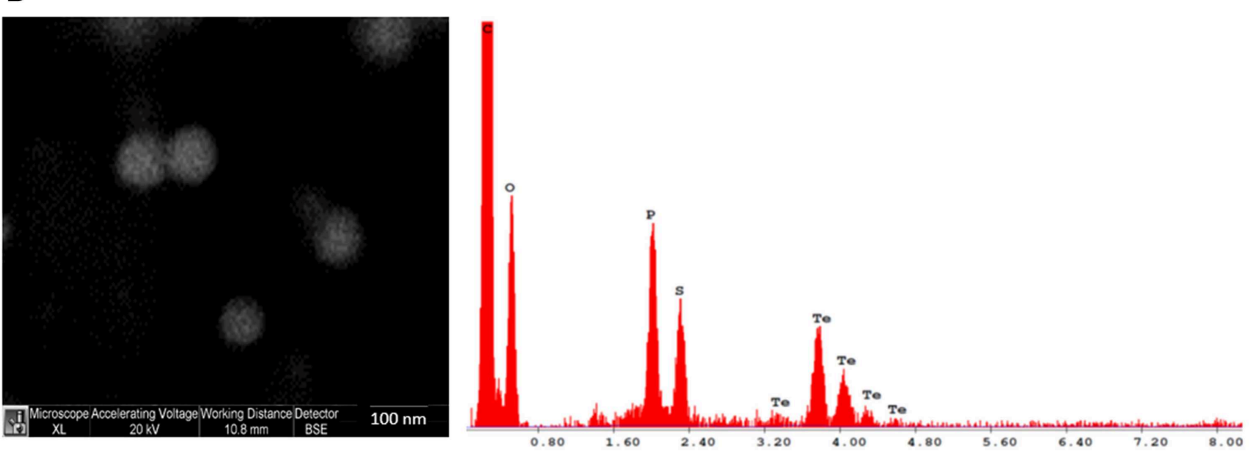

FIGURE 1 | Scanning electron microscopy (SEM) and energy dispersive X-ray (EDX) images of Se (A) and Te (B) NPs synthesized after 48 $\mathrm{h}$ of incubation.
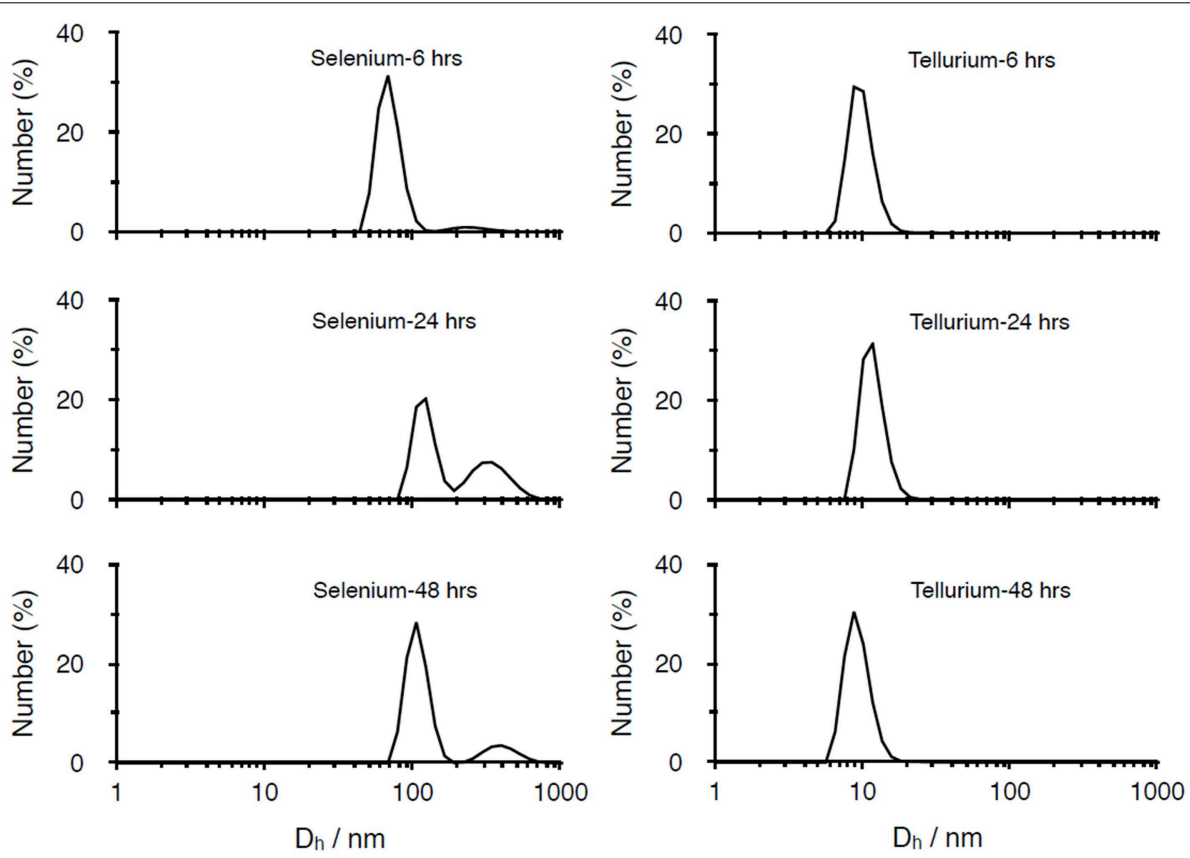

FIGURE 2 | DLS analysis of Se and Te-based nanoparticles produced after 6, 24, 48 h of incubation.

antimicrobial activities of NPs were compared with the toxic effect of the correspondent metal salt. Viable cell counts were determined for increasing NPs concentrations after $4 \mathrm{~h}$ of growth with NPs (to test their ability to inhibit biofilm formation) and $24 \mathrm{~h}$ of nanoparticles exposure of pre-established biofilms (to verify their biofilm-eradication activity). 
TABLE 1 | MIC (minimun inhibitory concentration) of Se and

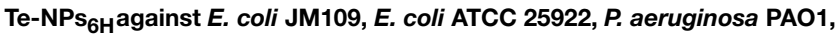
$P$. aeruginosa ATCC 27853, and S. aureus ATCC 25923.

\begin{tabular}{lcc}
\hline Strains & MIC SeNPs $_{\mathbf{6 H}} \mathbf{( m g / L )}$ & MIC TeNPs $_{\mathbf{6 H}} \mathbf{( m g / \mathbf { L } )}$ \\
\hline E. coli JM109 & 125 & 500 \\
E. coli ATCC 25922 & 125 & 500 \\
P. aeruginosa PAO1 & 250 & 500 \\
P. aeruginosa ATCC 27853 & 250 & 500 \\
S. aureus ATCC 25923 & 250 & 1000 \\
\hline
\end{tabular}

In Figure 3, the effect of $4 \mathrm{~h}$ exposure to SeNPs is summarized. In each one of the experiments, SeNPs exhibited the ability to kill $100 \%$ of both planktonic and biofilm populations. Moreover, $\mathrm{SeNPs}_{6 \mathrm{H}}$ and $\mathrm{SeNPs}_{24 \mathrm{H}}$ showed generally a higher antimicrobial and antibiofilm activity when compared with $\mathrm{SeNPs}_{48 \mathrm{H}}$ and selenite. For E. coli JM109 planktonic culture, we detected a toxic effect of SeNPs $6 \mathrm{H}$ and $\mathrm{SeNPs}_{24 \mathrm{H}}$ already at a concentration of $62.5 \mathrm{mg} / \mathrm{L}$ with a $3 \mathrm{Log}$ reduction. We achieve $100 \%$ kill of the planktonic culture at a SeNPs concentration of $125 \mathrm{mg} / \mathrm{L}$. A similar pattern was observed for the other two strains tested. For both $P$. aeruginosa PAO1 and $S$. aureus ATCC 25923, the concentration of $\mathrm{SeNPs}_{6 \mathrm{H}}$ and $\mathrm{SeNPs}_{24 \mathrm{H}}$ required to kill $100 \%$ of the planktonic population was $250 \mathrm{mg} / \mathrm{L}$. When we analyze the toxicity of SeNPs toward biofilm culture, we achieved the $100 \%$ kill of the culture at the same NPs concentration required to kill planktonic population, for both strains.

We can observe the toxic effect of $4 \mathrm{~h}$ exposure to TeNPs toward planktonic and biofilm cultures in Figure 4. In this case, tellurite exhibited the highest toxicity and different sized TeNPs showed similar toxicity between each other for the strain tested. For E. coli JM109 and P. aeruginosa PAO1, a concentration of $500 \mathrm{mg} / \mathrm{L}$ of TeNPs was required to kill $100 \%$ of planktonic cultures, while S. aureus ATCC 25923 culture was completely eradicated at a concentration of $1000 \mathrm{mg} / \mathrm{L}$. Biofilms were eradicated at the same TeNP concentrations that killed planktonic cultures, showing a similar resistance to TeNPs.

$\mathrm{EC}_{50}$ analysis (Figure 5) confirmed the higher toxicity of SeNPs $_{6 \mathrm{H}}$ and $\mathrm{SeNPs}_{24 \mathrm{H}}$ toward both planktonic and biofilm populations. For instance, $\mathrm{EC}_{50}$ of $\mathrm{SeNPs}_{6 \mathrm{H}}$ and $\mathrm{SeNPs}_{24 \mathrm{H}}$ for planktonic cultures of $E$. coli was respectively $26.32 \mathrm{mg} / \mathrm{L}$ and $30.51 \mathrm{mg} / \mathrm{L}$, while $\mathrm{EC}_{50}$ for $\mathrm{SeNPs}_{48 \mathrm{H}}$ was two time higher, $62.66 \mathrm{mg} / \mathrm{L}$. On the other hand, $\mathrm{EC}_{50}$ are similar between the different TeNPs tested, showing no influence of incubation time for TeNPs antimicrobial activity. Even in this case, for both SeNPs and TeNPs, biofilm cultures didn't exhibit a higher tolerance toward the antimicrobial action of the NPs when compare to planktonic populations.

\section{CLSM Analysis of Biofilm Cultures Exposed To Nanoparticles}

Another technique to characterize the antimicrobial effect of nanoparticles against biofilm culture is Confocal Laser Scanning Microscopy with LIVE/DEAD ${ }^{\circledR}$ BacLight $^{\mathrm{TM}}$ Bacteria Viability Kit (Life Technology). This staining uses two different dyes:
Syto9, a nucleic acid intercalator which fluoresce in green in living cells, and propidium iodide, which fluoresce in red after binding DNA or RNA of dead cells. By using this two dyes in conjunction, we obtain biofilm images in which viable cells are stained in green and dead cells in red.

After $24 \mathrm{~h}$ of growth in MBEC device, the strains formed a surface-adherent layer of bacterial cells with microcolonies of about $10-15 \mu \mathrm{m}$ in height.

As we can see from Figure 6 we can detect an effect of the NPs on biofilm structure for each one of the bacteria analyzed. At a concentration of $60 \mathrm{mg} / \mathrm{L}$, SeNPs completely eradicated the biofilm structure of $E$. coli. At the same concentration, SeNPs killed most part of biofilms cells of both $P$. aeruginosa and $S$. aureus, in contrast to growth control, formed for the most part of viable cells. On the other hand, at the same concentration of $60 \mathrm{mg} / \mathrm{L}$, TeNPs exhibited a lower toxicity effect toward biofilms structure: large portions of the biofilms are still composed by alive cells, in particular on the interior region of the structure. Nevertheless, biofilms in presence of TeNPs had a decreased thickness and less pronounced structural features.

These results confirm that biogenic NPs have antibiofilm activity against the three strains tested, as we have already seen with the viable cells count.

\section{Detection of Reactive Oxygen Species (ROS)}

In order to explore the mechanism of NPs toxicity, the production of reactive oxygen species (ROS) was evaluated in response to the NPs exposure. E. coli JM109, P. aeruginosa PAO1 and $S$. aureus ATCC 25923 were exposed for $3 \mathrm{~h}$ to the same concentration of NPs $(30 \mathrm{mg} / \mathrm{L})$ and reactive oxygen species (ROS) were quantified both in planktonic and biofilm cultures. Controls with different concentrations of both Se- and TeNPS were performed and we didn't detect interactions between nanoparticles and DCDFA (Figure S1).

In planktonic cultures, SeNPs induced a higher amount of ROS than selenite for each one of the strains tested $(P<0.05)$. Among the different SeNPs, there isn't a significant difference among nanoparticles produced after different incubation times. ROS production induced by TeNPs was, on the other hand, lower than that obtained after exposure to tellurite (Figure 7).

The same behavior was detected in biofilm cultures, with a similar amount of ROS generated.

\section{Discussion}

It is already well known that both metals and metalloids in their different ionic forms can exhibit a strong antimicrobial effect against microbial cells (Lemire et al., 2013). In recent years, however, a variety of nanostructured metals has evidenced very promising antibacterial properties. The uses of these metal nanoparticles have potential advantages over conventional antimicrobial agents due to their high surface to volume ratios that allow a higher area of interactions with biological systems. Nanomaterials also have unique physical and chemical properties that may lead to greater efficacy as a biocide. The present work shows that both biogenic $\mathrm{Se}^{0}$ and $\mathrm{Te}^{0}$ nanoparticles 


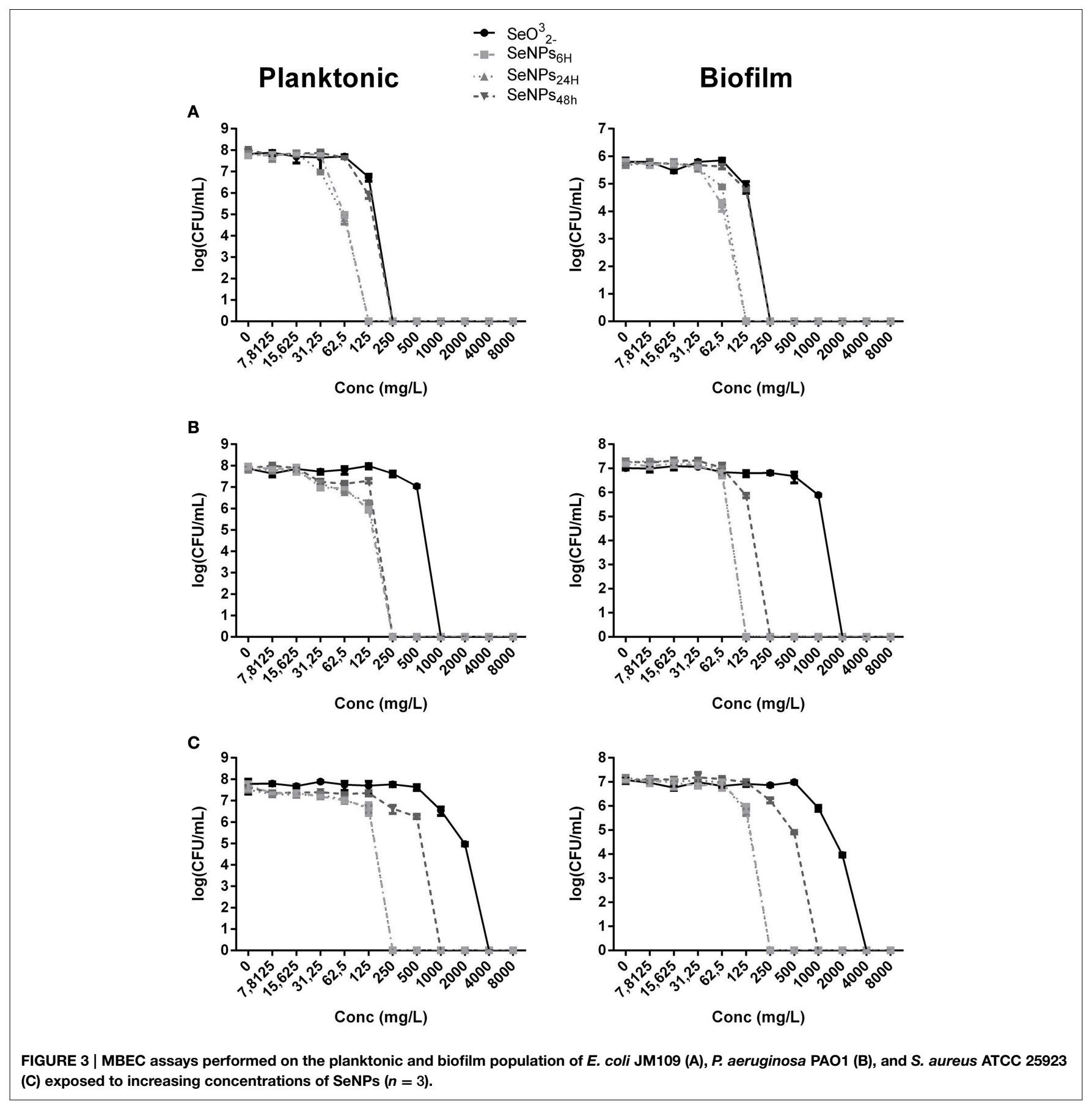

exhibited antimicrobial and anti-biofilm activity against E. coli, $P$. aeruginosa and $S$. aureus.

We report in the present study that biogenic SeNPs synthesized by the strain Stenotrophomonas maltophilia SeITE02 show characteristics analogous to those of previously described selenium nanoparticles of microbial origin. For instance, Lampis and coworkers reported that SeNPs from Bacillus mycoides SeITE01 owned spherical morphology and dimensions similar to the biogenic nanoparticles here discussed (Lampis et al., 2014). Indeed, a spherical morphology and dimensions between 100 and $400 \mathrm{~nm}$ are also typical of other biogenic SeNPs characterized elsewhere (Husen and Siddiqi, 2014).

On the other hand, there are far fewer descriptions on biogenic tellurium nanoparticles in literature. Zare and coworkers reported the purification of rod-shaped TeNPs from Bacillus sp. BZ (Zare et al., 2012). However, the nanoparticles produced by this strain evidenced significant differences with reference to either morphology or sizes compared to TeNPs analyzed in the present study. 
Zonaro et al.

Biogenic chalcogen nanoparticles as bactericides

Planktonic

A

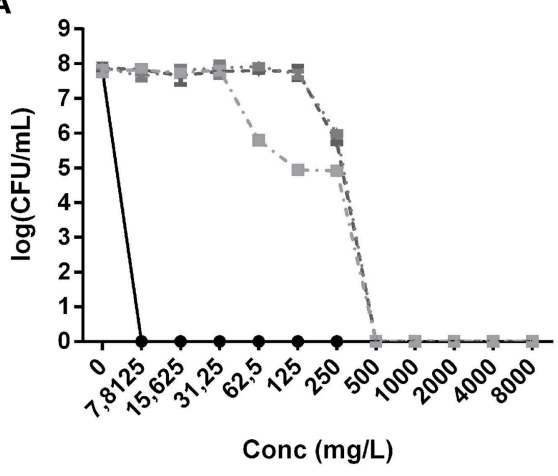

B

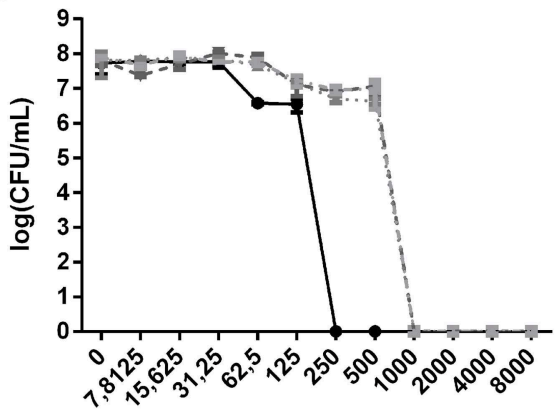

Conc (mg/L)

C

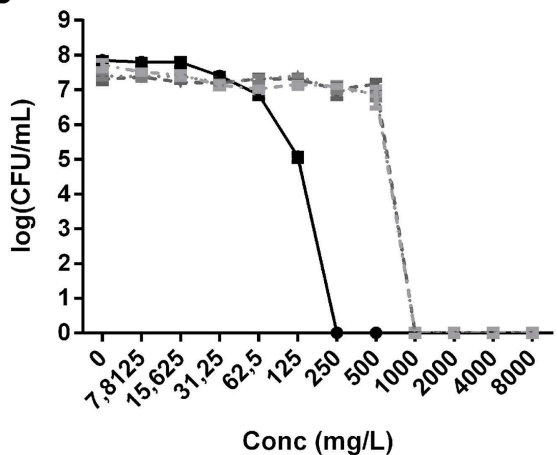

$\rightarrow \mathrm{TeO}_{2-}^{3}$

- TeNTs $_{6 \mathrm{H}}$

^. $\mathrm{TeNPs}_{24 \mathrm{H}}$

Biofilm

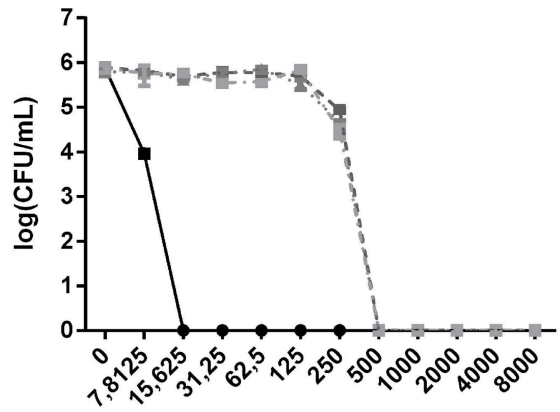

Conc (mg/L)

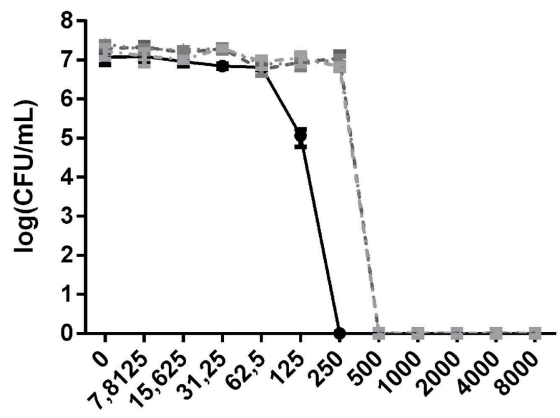

Conc (mg/L)

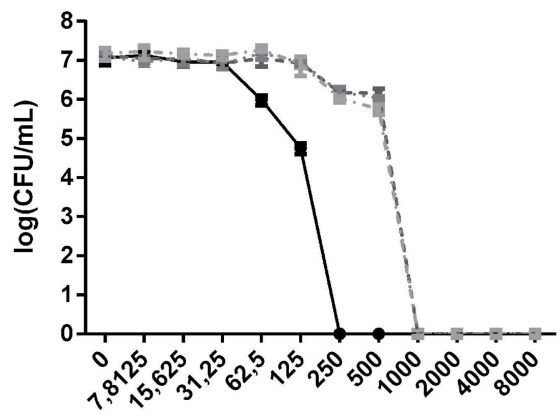

Conc (mg/L)

FIGURE 4 | MBEC assays performed on the planktonic and biofilm population of E. coli JM109 (A), P. aeruginosa PAO1 (B), and S. aureus ATCC 25923 (C) exposed to increasing concentrations of TeNDs $(n=3)$.

In our investigation, TeNDs generated by Ochrobactrum sp. MPV1 showed a lower antimicrobial activity than tellurite while SeNDs exerted a higher biocidal effect on microbial growth than selenite. This latter finding is surprising, since SeNDs have previously been reported to exhibit a lower toxicity with respect to the corresponding oxyanion, either in in vive or in vito tests (Shakibaie et al., 2013). Moreover, we demonstrated here that the antimicrobial activity of SeNDs shows a size-dependent response as already suggested by Lu et al. (2013). Smallest SeNDs ${ }_{6 \mathrm{H}}$, among the different dimensional classes of nanoparticles obtained in this study, exhibited the strongest antimicrobial activity with an $\mathrm{EC}_{50}$ of $26.32 \mathrm{mg} / \mathrm{L}$ for $E$. coli, $7.59 \mathrm{mg} / \mathrm{L}$ for $S$. aureus and $62.37 \mathrm{mg} / \mathrm{L}$ for $P$. aeruginosa, respectively, and the ability to completely inhibit biofilm formation at a concentration of $125 \mathrm{mg} / \mathrm{L}$ for E. coli and P. aeruginosa and $250 \mathrm{mg} / \mathrm{L}$ for $S$. aureus. As nanoparticles decrease in size, their surface to volume ratio increase, confirming smaller is better for improving the biological reactivity. Nevertheless, size is not the sole parameter influencing antimicrobial properties of the nanoparticles: other important features are both the elemental composition and the shape $(\mathrm{Pal}$ et al., 2007). In this respect, for the antimicrobial activity of TeNDs, we did not detect a significant difference between RPs

Frontiers in Microbiology | www.frontiersin.org

7

June 2015 | Volume 6 | Article 584 
4 hours exposure

A

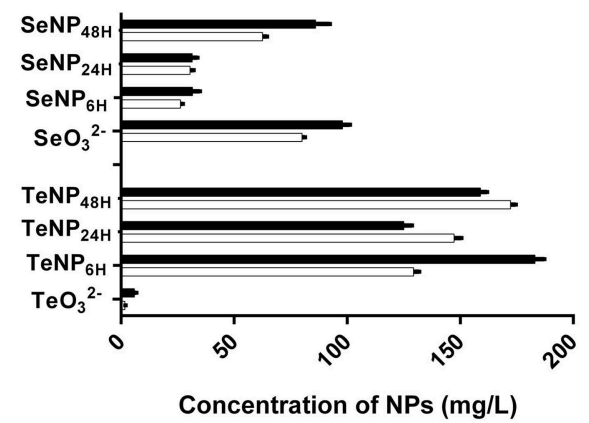

B

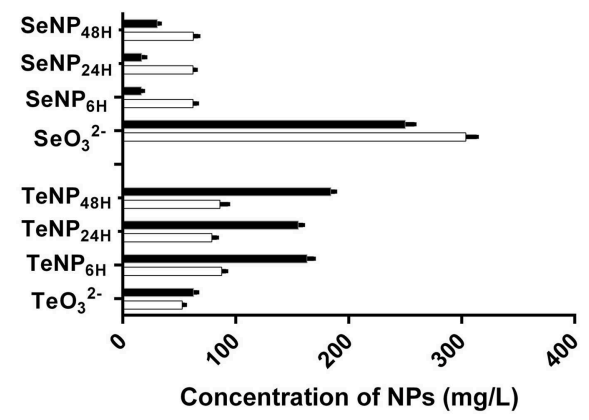

C

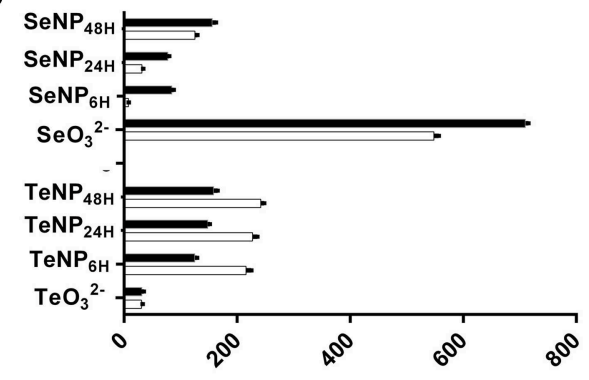

Concentration of NPs (mg/L)

\section{4 hours growth + 24 hours exposure}
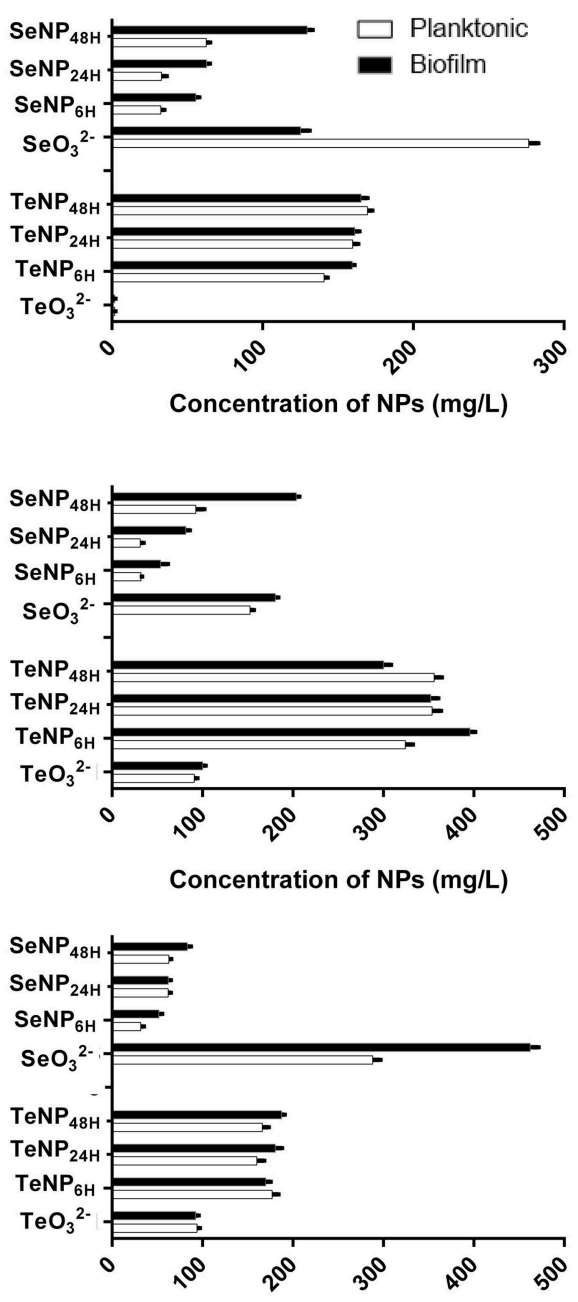

Concentration of NPs (mg/L)

FIGURE 5 | EC 50 of SeNPs and TeNPs for planktonic and biofilm cultures of E. coli JM109 (A), P. aeruginosa PAO1 (B), and S. aureus ATCC 25923 (C) after $4 \mathrm{~h}$ of exposure and $24 \mathrm{~h}$ of growth $+24 \mathrm{~h}$ of exposure.

produced at different incubation times (leading to different sizes). The $\mathrm{EC}_{50}$ values of differently sized TeNPs were $160 \mathrm{mg} / \mathrm{L}$ for $E$. coli, $150 \mathrm{mg} / \mathrm{L}$ for S. aureus, and $175 \mathrm{mg} / \mathrm{L}$ for $P$. aeruginosa.

Antimicrobial activity of various biogenic SeNPs and TeNPs has been explored before, however, different methodologies were used to evaluate the bactericidal activity. Although particles and protocols are different, the resistance levels reported in literature are similar to the concentrations found in this work. In particular, SeNPs produced by Bacillus sp. MSh-1 resulted in the inhibition of biofilm formation by $S$. aureus ATCC 25923, P. aeruginosa, and $P$. mirabilis already at a concentration of $16 \mathrm{mg} / \mathrm{L}$, with a decrease of biofilm formation of $58 \%$ for S. aureus, $65.7 \%$ for $P$. aeruginosa and $46.6 \%$ for $P$. mirabilis (Shakibaie et al., 2015). Chemically synthesized SeNPs have also been reported as capable of antimicrobial activity. Tran and Webster studied the effect of SeNPs on S. aureus ATCC 25923. Their observations indicate that SeNPs cause a $3 \log$ inhibition of $S$. aureus growth at concentrations between 7.8 and $31 \mathrm{mg} / \mathrm{L}$ after $3 \mathrm{~h}$ of exposure (Tran and Webster, 2011). Moreover, Chudobova et al. reported their chemically synthesized SeNPs with a higher antimicrobial activity than AgNPs, inducing a complete growth inhibition of $S$. aureus NCTC 8511 at a concentration of $23.7 \mathrm{mg} / \mathrm{L}$ (Chudobova et al., 2014).

Biogenic TeNPs have also been studied for their potential bactericidal activity. Zare et al. (2012) demonstrated antibacterial activity of TeNPs against different clinical isolates (S. aureus, $P$. aeruginosa, S. typhi and K. pneumonia), with an MBC (minimum bactericidal concentration) between 125 and 


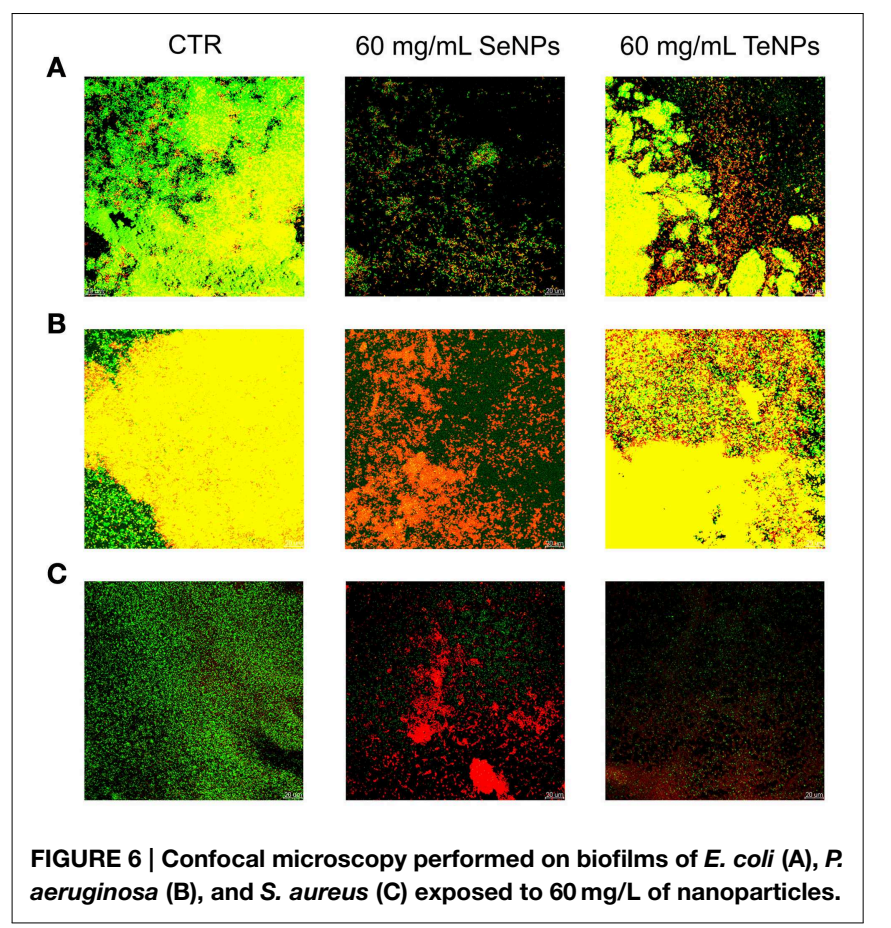

$500 \mathrm{mg} / \mathrm{L} \quad$ (Zare et al., 2012). Nevertheless, by comparing the antimicrobial activity of SeNPs and TeNPs with silver nanoparticles (AgNPs), these latter generally show antimicrobial activity at lower concentrations than both SeNPs and TeNPs. In fact, AgNPs completely inhibited biofilm formation in E. coli $\mathrm{AB} 1157$ and $P$. aeruginosa PAO1 at a concentration of 5 and $10 \mathrm{mg} / \mathrm{L}$, respectively. However, the concentration required to eradicate an already formed biofilm structure is over $150 \mathrm{mg} / \mathrm{L}$ (Radzig et al., 2013), similar to the values reported in the present study for biogenic SeNPs and TeNPs.

An unexpected finding in the present investigation is that both the biogenic SeNPs and TeNPs exhibited equivalent antimicrobial efficacy toward the bacterial cells grown either in plankonic form or in biofilm mode. This evidence is particularly significant since bacteria grown in biofilm form are reported to be more tolerant to antimicrobial agents, in particular to traditional antibiotics (Hall-Stoodley et al., 2004).

On the basis of both the evaluation of antimicrobial activity and CLSM observations, it appears evident that SeNPs and TeNPs possess bactericidal properties. Anyway, the mechanisms responsible for the antimicrobial activity are not yet completely understood. One of the possible modes of action that has been proposed to explain the toxicity of other nanomaterials

\section{$\mathrm{Se}^{0}$}

$\mathrm{Te}^{0}$

A

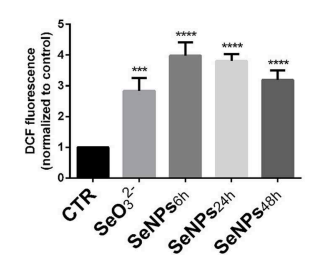

B

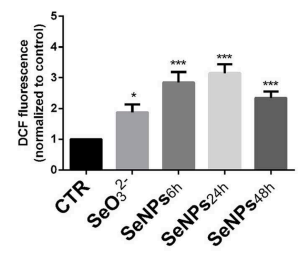

C

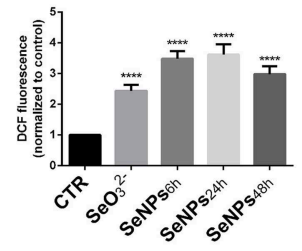

Biofilm
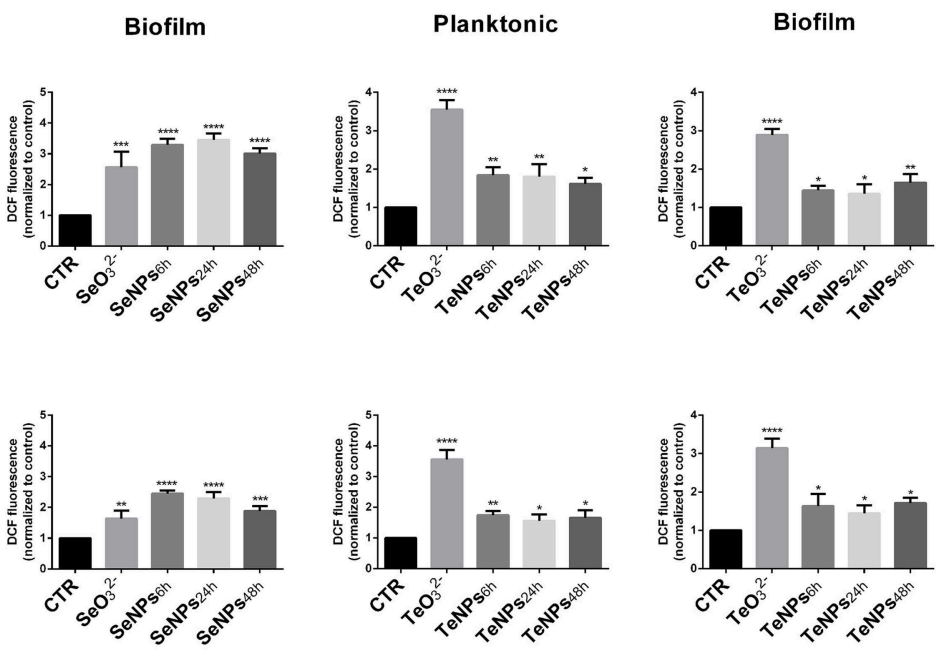

Biofilm

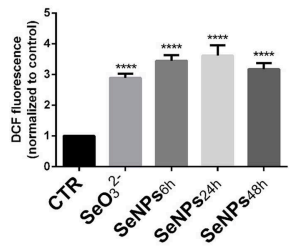

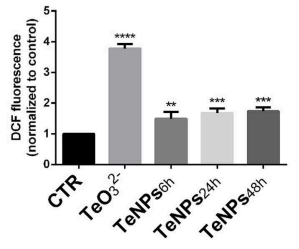

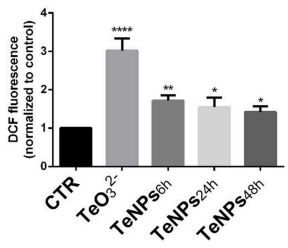

FIGURE 7 | Formation of ROS in E. coli (A), P. aeruginosa (B), and S. aureus (C) cells exposed to $30 \mathrm{mg} / \mathrm{L}$ of SeNPs and TeNPs. ( $n=3 ; P<0.05)$. 
(AgNPs and $\mathrm{ZnNPs}$ ) is the production of reactive oxygen species (ROS) (Manke et al., 2013). Reports already exist in the literature that attribute the antibacterial effects of different selenium compounds to the formation of free radicals (Tran et al., 2009). Moreover, selenium and tellurium oxyanions have also been found to trigger the generation of ROS, with both elements capable of reacting with intracellular thiols and forming intermediates that cause oxidative stress as a consequence of the formation of superoxide radicals (Zannoni et al., 2008). In the present study, after TeNPs exposition, the amount of ROS induced is lower than that recorded after tellurite exposure. On the other hand, we observed that SeNPs induce a higher production of ROS compared to selenite. However, we did not observe induction of ROS formation by SeNPs in a sizedependent manner. In fact, the amounts of ROS generated by different dimensional types of SeNPs were very similar, even if the toxicity effects they exert to the microbial populations increase with the lowest dimensions. Thus, even if reactive oxygen species are involved in the toxicity of NPs, there must be other mechanisms responsible for the antimicrobial activity of these nanostructured metals. For instance, it seems that nanoparticles can contribute to functional damages of cell membrane or wall by disrupting the integrity of these important envelopes (Pi et al., 2013). Some other mechanism related to the surface features of the nanoparticles may be involved however in conferring toxicity to NPs (Bao et al., 2015).

In conclusion, the study presented here concerns the analysis of the antibacterial properties of elemental selenium and tellurium nanoparticles of biogenic origin against reference strains of biofilm-forming bacteria tested either in planktonic cultures or as biofilms. Both $\mathrm{Se}^{0}$ and $\mathrm{Te}^{0}$ nanoparticles have demonstrated to efficaciously inhibit planktonic and biofilm growth of E. coli JM109, P. aeruginosa PAO1, and S. aureus ATCC 25923. It is worth noting that these nanoparticles

\section{References}

Bao, P., Chen, Z., Tai, R. Z., Shen, H. M., Martin, F. L., and Zhu, Y. G. (2015). Selenite-Induced toxicity in cancer cells is mediated by metabolic generation of endogenous selenium nanoparticles. J. Proteome Res. 14, 1127-1136. doi: $10.1021 /$ pr501086e

Chudobova, D., Cihalova, K., Ruttkay-Nedecky, B., Merlos Rodrigo, M. A., Tmejova, K., Kopel, P., et al. (2014). Comparison of the effects of silver phosphate and selenium nanoparticles on Staphylococcus aureus growth reveals potential for selenium particles to prevent infection. FEMS Microbiol. Lett. 351, 195-201. doi: 10.1111/1574-6968.12353

Di Gregorio, S., Lampis, S., and Vallini, G. (2005). Selenite precipitation by a rhizospheric strain of Stenotrophomonas sp. isolated from the root system of Astragalus bisulcatus: a biotechnological perspective. Environ. Int. 31, 233-241. doi: 10.1016/j.envint.2004.09.021

Fabrega, J., Renshaw, J. C., and Lead, J. R. (2009). Interactions of silver nanoparticles with Pseudomonas putida biofilms. Environ. Sci. Technol. 43, 9004-9009. doi: 10.1021/es901706j

Hall-Stoodley, L., Costerton, J., and Stoodley, P. (2004). Bacterial biofilms: from the natural environment to infectious diseases. Nat. Rev. Microbiol. 2, 95-108. doi: $10.1038 /$ nrmicro821

Harrison, J. J., Stremick, C. A., Turner, R. J., Allan, N. D., Olson, M. E., and Ceri, H. (2010). Microtiter susceptibility testing of microbes growing on peg lids: a miniaturized biofilm model for high- evidenced the ability to hinder biofilm formation and, likewise, to completely eradicate already established biofilms. In particular, antimicrobial activity of SeNPs is size dependent. Another element of absolute novelty is that $\mathrm{Se}^{0}$ nanoparticles showed a higher antimicrobial activity than the free oxyanion selenite $\left(\mathrm{SeO}_{3}^{2-}\right)$ well recognized to be quite toxic to biota. Indeed, previous works on antimicrobial activity of metal nanoparticles reported that anionic forms are usually more toxic to bacteria than the corresponding nanoparticles. The evidence that $\mathrm{Se}^{0}$ and $\mathrm{Te}^{0}$ nanoparticles-obtained through the reduction of selenite and tellurite by Stenotrophomonas maltophilia SeITE02 and Ochrobactrum sp. MPV1, respectively-are capable of inducing the formation of ROS as possible cause of microbial growth inhibition deserves also particular attention. Recently, the activity of a different form of biogenic $\mathrm{Se}^{0}$ nanoparticles was explored against biofilm cultures (Shakibaie et al., 2015).

The evidence gained in this study and the results of other recent investigations strengthen the perspective of a possible use of SeNPs and TeNPs as efficacious antimicrobial agents.

\section{Acknowledgments}

This work was supported by the Natural Science and Engineering Research Council of Canada (NSERC) and Canadian Institutes of Health and Research (CIHR) to RT. The financial support of the Hungarian-Italian intergovernmental scientific and technological cooperation program (TET_10-1-2011-0173 and PGR_00086-2011) is gratefully acknowledged.

\section{Supplementary Material}

The Supplementary Material for this article can be found online at: http://journal.frontiersin.org/article/10.3389/fmicb. 2015.00584/abstract throughput screening. Nat. Protoc. 5, 1236-1254. doi: 10.1038/nprot. 2010.71

Husen, A., and Siddiqi, K. S. (2014). Plants and microbes assisted selenium nanoparticles: chacterization and application. J. Nanobiotechnol. 12:28. doi: 10.1186/s12951-014-0028-6

Jones, N., Ray, B., Ranjit, K. T., and Manna, A. C. (2008). Antibacterial activity of $\mathrm{ZnO}$ nanoparticles suspensions on a broad spectrum of microorganisms. FEMS Microbiol. Lett. 279, 71-76. doi: 10.1111/j.1574-6968.2007.01012.x

Lampis, L., Zonaro, E., Bertolini, C., Bernardi, P., Butler, C. C., and Vallini, G. (2014). Delayed formation of zero-valent selenium nanoparticles by Bacillus mycoides SeITE01 as a consequence of selenite reduction under aerobic conditions. Microb. Cell Fact. 13, 35-49. doi: 10.1186/1475-2859-13-35

Lemire, J. A., Harrison, J. J., and Turner, R. J. (2013). Antimicrobial activity of metals: mechanisms, molecular targets and applications. Nat. Rev. Microbiol. 11, 371-384. doi: $10.1038 /$ nrmicro3028

Lu, Z., Rong, K., Li, J., Yang, H., and Chen, R. (2013). Size-dependent antibacterial activities of silver nanoparticles against oral anaerobic pathogenic bacteria. $J$. Mater. Sci. Mater. Med. 24, 1465-1471. doi: 10.1007/s10856-013-4894-5

Manke, A., Wang, L., and Rojanasakul, Y. (2013). Mechanisms of nanoparticles induced oxidative stress and toxicity. Biomed. Res. Int. 2013:942916. doi: $10.1155 / 2013 / 942916$

Martinez-Gutierrez, F., Boegli, L., Agostinho, A., Sanchez, E. M., Bach, H., Ruiz, F., et al. (2013). Anti-biofilm activity of silver nanoparticles against different microorganisms. Biofouling 29, 651-660. doi: 10.1080/08927014.2013.794225 
Mohanty, A., Kathawala, M. H., Zhang, J., Chen, W. N., Loo, J. S. C., Kjelleberg, S., et al. (2014). Biogenic tellurium nanorods as a novel antivirulence agent inhibiting pyoverdine production in Pseudomonas aeruginosa. Biotechnol. Bioeng. 111, 858-865. doi: 10.1002/bit.25147

Narayanan, K. B., and Sakthivel, N. (2010). Biological synthesis of metal nanoparticles by microbes. Adv. Colloid Interface Sci. 156, 1-13. doi: 10.1016/j.cis.2010.02.001

Pi, J., Yang, F., Jin, H., Huang, X., Liu, R., Yang, P., et al. (2013). Selenium nanoparticles induced membrane bio-mechanical property changes in MCF-7 cells by disturbing membrane molecules and F-actin. Bioorg. Med. Chem. Lett. 23, 6296-6303. doi: 10.1016/j.bmcl.2013.09.078

Pal, S., Tak, Y. K., and Song, J. M. (2007). Does the antibacterial activity of silver nanoparticles depend on the shape of the nanoparticles? A study of the gramnegative bacterium Escherichia coli. Appl. Environ. Microbiol. 73, 1712-1720. doi: 10.1128/AEM.02218-06

Radzig, M. A., Nadtochenko, V. A., Koksharova, O. A., Kiwi, J., Lipasova, V. A., and Khmel, I. A. (2013). Antibacterial effects of silver nanoparticels on gram-negative bacteria: influence on the growth and biofilm formation, mechanisms of action. Colloids Surf. B Biointerfaces 102, 300-306. doi: 10.1016/j.colsurfb.2012.07.039

Roe, D., Karandikar, B., Bonn-Savage, N., Gibbins, B., and Roullet, J. B. (2008). Antimicrobial surface functionalization of plastic catheters by silver nanoparticles. J. Antimicrob. Chemother. 61, 869-876. doi: 10.1093/jac/dkn034

Santi, C. (2013). The Interaction between Rhizobacteria and the Hyperaccumulator Fern Pteris Vittata in Arsenic Transformation. Doctoral thesis, University of Verona.

Shakibaie, M., Shahverdi, A. R., Faramarzi, M. A., Hassanzadeh, G. R., Rahimi, H. R., et al. (2013). Acute and subacute toxicity of novel biogenic selenium nanoparticles in mice. Pharm. Biol. 51, 58-63. doi: 10.3109/13880209.2012.710241

Shakibaie, M., Forootanfar, H., Golkari, Y., Mohammadi-Khorsand, T., and Shakibaie, M. R. (2015). Anti-biofilm activity of biogenic selenium nanoparticles and selenium dioxide against clinical isolates of Staphylococcus aureus, Pseudomonas aeruginosa, and Proteus mirabilis. J. Trace Elem. Med. Biol. 29, 235-241. doi: 10.1016/j.jtemb.2014.07.020

Sievert, D. M., Ricks, P., Edwards, J. R., Schneider, A., Patel, J., Srinivasan, A., et al. (2013). Antimicrobial-resistant pathogens associated with healthcareassociated infections: summary of data reported to the national healthcare safety network at the centers for disease control and prevention, 2009-2010. Infect. Control Hosp. Epidemiol. 31, 1-14. doi: 10.1086/668770
Sondi, I., and Salopek-Sondi, B. (2004). Silver nanoparticles as antimicrobial agent: a case study on E. coli as model for Gram-negative bacteria. J. Colloid Interface Sci. 275, 177-182. doi: 10.1016/j.jcis.2004.02.012

Stewart, P. S., and Costerton, J. W. (2001). Antibiotic resistance of bacteria in biofilms. Lancet 358, 135-138. doi: 10.1016/S0140-6736(01)05321-1

Tran, P. L., Hammond, A. A., Mosley, T., Cortez, J., Gray, T., Colmer-Hamood, J. A., et al. (2009). Organoselenium coating on cellulose inhibits the formation of biofilms by Pseudomonas aeruginosa and Staphylococcus aureus. Appl. Environ. Microbiol. 75, 3586-3592. doi: 10.1128/AEM.02683-08

Tran, P. A., and Webster, T. J. (2011). Selenium nanoparticles inhibit Staphylococcus aureus growth. Int. J. Nanomedicine 6, 1553-1558. doi: 10.2147/IJN.S21729

Tsuang, Y. H., Sun, J. S., Huang, Y. C., Lu, C. H., Chang, W. H., and Wang, C. C. (2008). Studies of photokilling of bacteria using titanium dioxide nanoparticles. Artif. Organs. 32, 167-174. doi: 10.1111/j.1525-1594.2007.00530.x

Wang, H., and Joseph, J. A. (1999). Quantifying cellular oxidative stress by dichlorofluorescein assay using microplate reader. Free Rad. Bio. Med. 27, 612-616. doi: 10.1016/S0891-5849(99)00107-0

Zannoni, D., Borsetti, F., Harrison, J. J., and Turner, R. J. (2008). The bacterial response to the chalcogen metalloids Se and Te. Adv. Microb. Physiol. 53, 1-72. doi: 10.1016/S0065-2911(07)53001-8

Zare, B., Faramarzi, M. A., Sepehrizadeh, Z., Shakibaie, M., Rezaie, S., and Shahverdi, A. R. (2012). Biosynthesis and recovery of rod-shaped tellurium nanoparticles and their bactericidal activities. Mat. Res. Bull. 47, 3719-3725. doi: 10.1016/j.materresbull.2012.06.034

Zhang, M., Zhang, K., De Gusseme, B., and Verstarete, W. (2012). Biogenic silver nanoparticles (bio-Ag 0) decrease biofouling of bio-Ag 0/PES nanocomposite membranes. Water Res. 46, 2077-2087. doi: 10.1016/j.watres.2012. 01.015

Conflict of Interest Statement: The authors declare that the research was conducted in the absence of any commercial or financial relationships that could be construed as a potential conflict of interest.

Copyright (c) 2015 Zonaro, Lampis, Turner, Qazi and Vallini. This is an open-access article distributed under the terms of the Creative Commons Attribution License (CC $B Y)$. The use, distribution or reproduction in other forums is permitted, provided the original author(s) or licensor are credited and that the original publication in this journal is cited, in accordance with accepted academic practice. No use, distribution or reproduction is permitted which does not comply with these terms. 